\title{
Issues with body mass measurement on the International Space Station (ISS)
}

\author{
Kazuhito Shimada ${ }^{1,}$, , Yusaku Fujii ${ }^{2, b}$ \\ ${ }^{1}$ Medical Operations, JAXA Tsukuba Space Center, 2-1-1 Sengen, Tsukuba 305-8505 Japan \\ ${ }^{2}$ Department of Electronic Engineering, Gunma University, Kiryu 376-8515, Japan \\ ashimada.kazuhito@jaxa.jp, ${ }^{b}$ fujii@el.gunma-u.ac.jp
}

Keywords: Body Mass, Body Weight, International Space Station, Skylab, Mass Measurement, Astronaut, Metrology, Drop Tower

Abstract. Although inception of the measurement of astronaut body mass measurement was as old as Skylab era, progress in the field has not been as expected. There are fundamental physical difficulties as well as program management issues. New mass measurement systems with the current sensor technologies should be prepared, and the new sensor system could be expanded for "Drop Tower" in space.

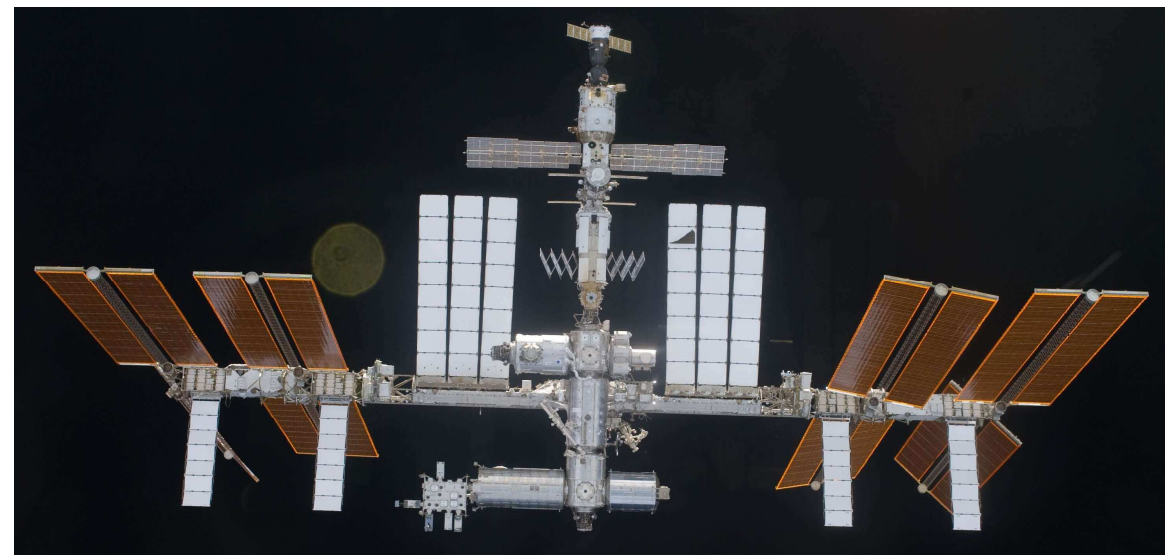

Fig. 1. International Space Station (ISS).

ISS photographed from approaching Space Shuttle STS-132 on 16 May 2010. Bottom left are Japanese Kibo/JEM modules. Bottom right is Columbus module from ESA. Upper half are Russian modules. NASA modules are in the center area. Large truss supports four large solar paddles and radiators.

\section{Introduction}

This is the era of International Space Station (ISS), whose first element was launched in 1998 (Fig.1). Its assembly is concluding in 2010, but its expansion and renewal of modules, as well as onboard experiment hardware, will continue until its systems service life of 2028, or International Partner (IP) agreement date. IP's include USA/NASA, Russia/FSA, Japan/JAXA, Europe/ESA, and Canada/CSA.

The history of space station is not short. It started with the Russian space station Salyut in 1971 (Fig.2). Then US Skylab was launched in 1973. The Skylab had 3 sets of crew during its service life. The name of Salyut-3 was given to the Russian Almaz space station system in 1974. The Russian space station was upgraded to a very complex Mir space station, starting in 1986. It started as a fairly simple system, but eventually evolved to a complex Russia-US joint space station. The latter joint stage of the Mir operation period was called Space Station Phase 1 or Shuttle-Mir program by NASA.

Because of the long duration stay of astronauts/cosmonauts, body mass management for astronaut health became an operational concern as well as science demand [1,2]. 


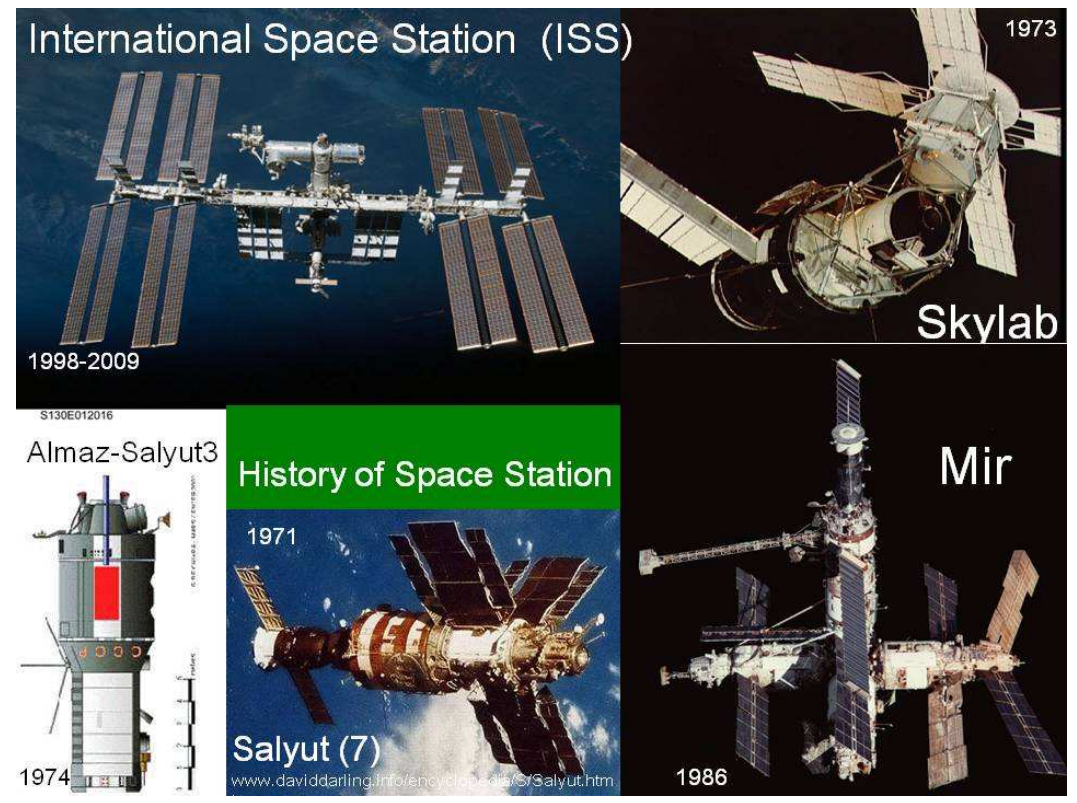

Fig.2. History of Space Stations. Russian Salyut space station was the first space station. Bearing the same name of Salyut was the Almaz space system, which was different from other Salyut stations. Its hardware is said to contain military applications, like a space gun. Almaz system is planned to be a basis for commercial space transportation system. US/NASA Skylab was launched in 1973 and housed 3 sets of crew. Mir space station, launched in 1986, had mechanism to expand. It evolved into a multi-module complex space station. Photos: NASA, FSA, www.daviddarling.info.

Japan Aerospace Exploration Agency (JAXA) sent two astronauts onboard ISS (Fig.3). The first completed a four-and-a-half month stay, and the second is to finish a six-month mission. They were also subjects for monthly body mass measurement.

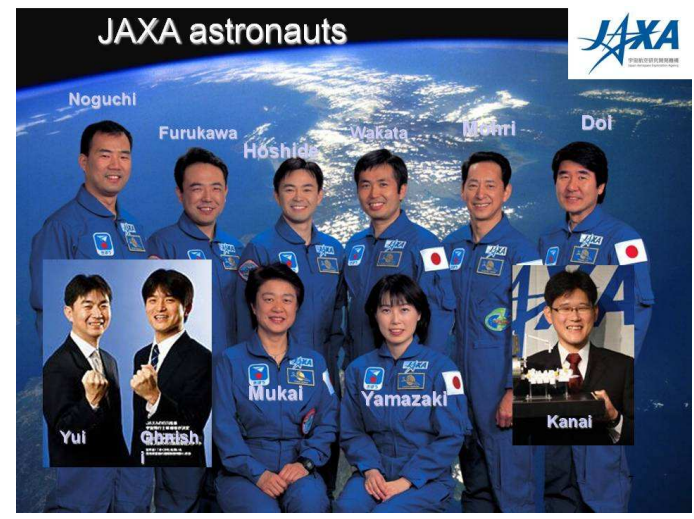

Fig.3. Japan Aerospace Exploration Agency (JAXA) astronauts.

Out of 8 astronauts, 2 flew on ISS. The other 6 made short duration flights aboard the Space Shuttle. Three are under training as astronaut candidates.

Photo: www.jaxa.jp

\section{Performance Requirement of Body Mass Measurement Device}

First, we have to know what "body mass" means. As we are to discuss measurement on orbit, we have to think about 'mass' rather than 'weight'. The ISS acceleration environment has residual ' $G$ ' level of around $10^{-5} \mathrm{G}$, that does not affect body mass measurement. Here, ' $1 \mathrm{G}$ ' means acceleration on the surface of the Earth, i.e. $9.8 \mathrm{~m} \mathrm{~s}^{-2}$.

Then how much precision do we need to measure body mass? Body mass is not a constant through a day. As shown in Fig. 4 for a typical case for a 70kg person, it varies due to fluid dynamics of the body.

$\begin{array}{ll}\text { INPUT } & \sim 1500 \mathrm{~mL} \\ \text { drink water } & \sim 1000 \mathrm{~mL} \\ \text { food water } & \sim 300 \mathrm{~mL} \\ \text { food metabolic water } & \\ \text { OUTPUT } & 300 \mathrm{~mL} \\ \text { breath water } & 900 \mathrm{~mL} \\ \text { non-droplet perspiration } & 100 \mathrm{~mL} \\ \text { stool } & \sim 1500 \mathrm{~mL} \\ \text { urine } & \end{array}$

Fig.4. Typical daily water balance for a human body.

$70 \%$ of body mass is water. The figures are for typical $70 \mathrm{~kg}$ person's water balance within a day. 
From the water balance numbers in Fig.4, we can specify a useful and meaningful precision for body mass measurement, as in Table 1.

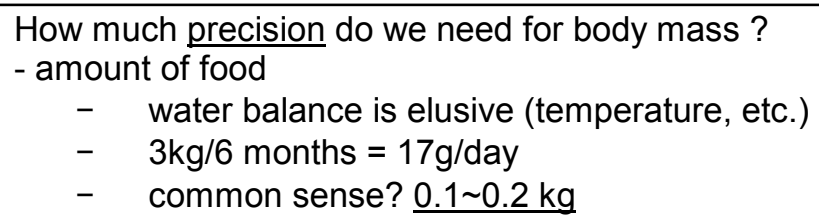

Table 1. Precision for body mass measurement.

Useful precision for body mass measurement are inferred from numbers in Fig.4.

Mid- to long-term body mass change is affected by intake calories and amount of exercise. Food-calorie relationship is shown in Table 2. A body mass measurement system is expected to detect change in nutritional status. An example of ISS astronaut exercise is found in Fig. $\mathbf{5}$.
ISS astronauts exercise $\sim 2 \mathrm{~h} /$ day.
- fat $9 \mathrm{kcal} / \mathrm{g}$, carbohydrate or protein $4 \mathrm{kcal} / \mathrm{g}$.
- combined food calorie $\sim 6 \mathrm{kcal} / \mathrm{g}, 500 \mathrm{~g} / 3,000 \mathrm{kcal}$.
- $1.5 \mathrm{~h} @ 200 \mathrm{kcal} / \mathrm{h}=300 \mathrm{kcal}=33 \mathrm{~g}$ of fat

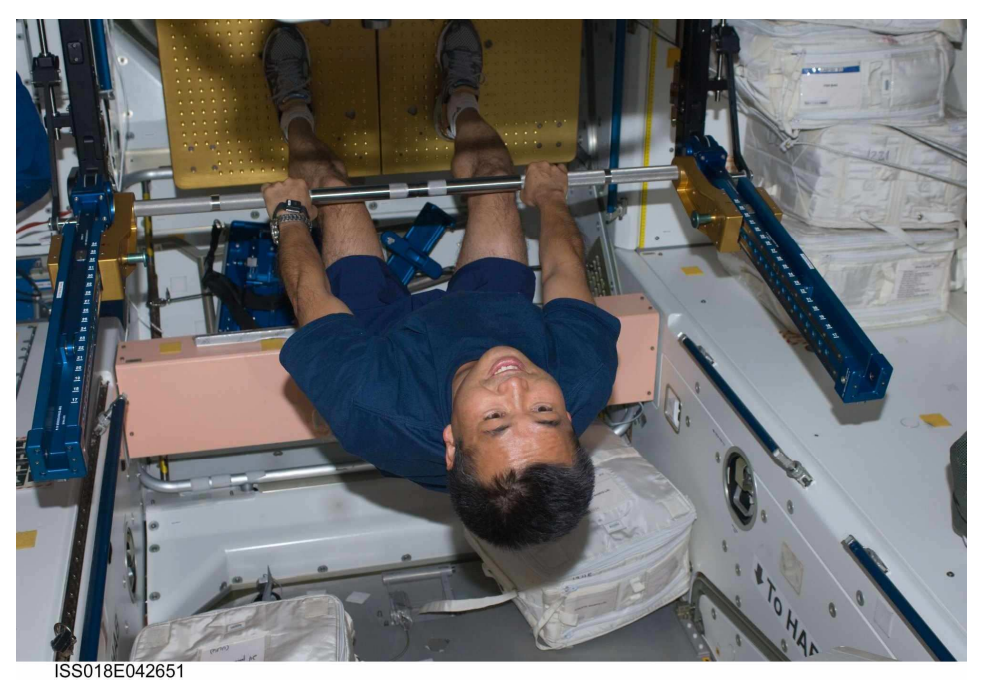

Table 2. Calorie intake, exercise and body mass change.
Fig.5 . Exercise onboard ISS.

ISS Expedition 18 astronaut Koichi Wakata/JAXA is using Advanced Resistive Exercise Device, "ARED". Performing upper body strengthening pull-ups in the Unity node of the International Space Station. (22 March 2009) Photo: NASA.

\section{Nutritional Variance}

The most influential factor to astronaut body mass change is nutrition [3]. The reduction of body mass in the first week of orbital stay is attributed to fluid shift effect, but the rest of the body mass change, mostly reduction, is due to nutrition. Tracking the exact input and output was conducted on Skylab missions. On ISS, intake is estimated by Food Frequency Questionnaire on ISS laptop computer (Fig. 6, Fig.7). Output (i.e. urine and stool) mass is not measured as on Skylab, thus body mass numbers are important for health care. 


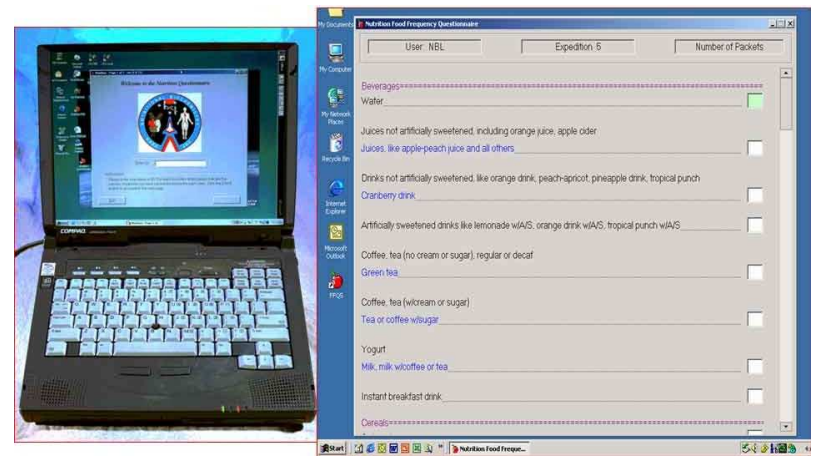

Fig. 6. ISS Food Frequency Questionnaire system.

Astronauts onboard ISS fills out food questions once every week. From these data, calorie and other nutritional element intake are estimated [3].

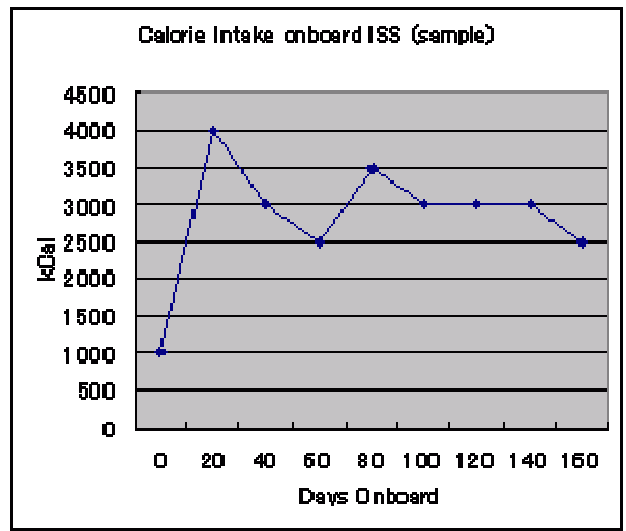

Fig. 7. Sample Food Frequency Questionnaire result.

From weekly survey, calorie intake per day is estimated for each ISS crewmember. Here is a sample of calorie intake per day. Long duration crewmembers eat less in the first week of stay due to space adaptation symptoms, which include less appetite. They exercise regularly, about two hours everyday, following daily schedule.

ISS space food is supplied by US, Russia, Japan, and Europe. In spite of diversified menu, crewmembers tend to eat less than recommended. Daily exercise consumes more than usual energy. Result is reduced body mass among almost all crew, except for later Skylab missions (Fig.8).

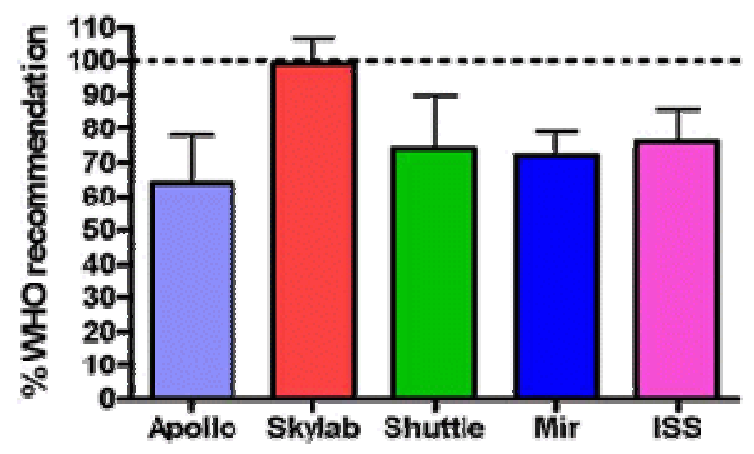

Fig. 8. Astronauts eat less than ideal food intake.

Besides later Skylab missions, almost all NASA astronauts ate less than recommended calorie [3].

How Skylab was different in space food? Their food was well prepared (Fig.9), they had a refrigerator for food, and recording nutritional state was one of mission objectives. The meal plan was tested and revised on the ground with "SMEAT" long duration simulation mission before Skylab flight mission $[1,2]$.

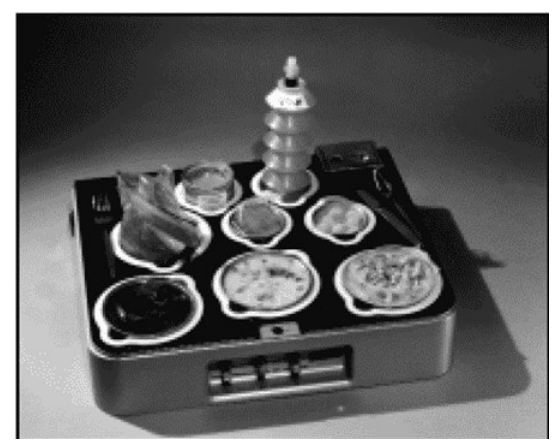

Fig. 9. Skylab meal tray.

The supply of food on Skylab was sufficient to feed 3 astronauts for approximately 112 days. Each astronaut's caloric intake was 2,800 kcal/day. Nutritional requirements were part of the life science experiments conducted on Skylab. Everybody onboard was a subject of the experiment.

Photo: NASA EG-1999-02-115-HQ 


\section{Principles of Mass Measurement on Spacecraft}

Because of lack of steady acceleration field within space vehicles, ground use load cells or balances are not operable on ISS. Physical principles for mass measurement actually applied were (Fig.10):

1) mass-spring resonance period (Fig.11)

(Skylab, Space Shuttle - Spacelab, Mir, and ISS MO-8 BMMD)

2) spring force and acceleration sensor (ISS SLAMMD)

Other principles proposed are:

1) centrifugal force (proposed for ISS specimen mass device [4])

2) momentum change against a reference mass (Levitation Mass Method [5])

3) linear acceleration and direct force sensor (Space Scale $[6,7]$ )

As there has been no spacecraft with artificial gravity, use of load cells or balances on spacecraft has not been proposed, until Thornton proposed "ISS Drop Tower" using expanded version of Space Scale.

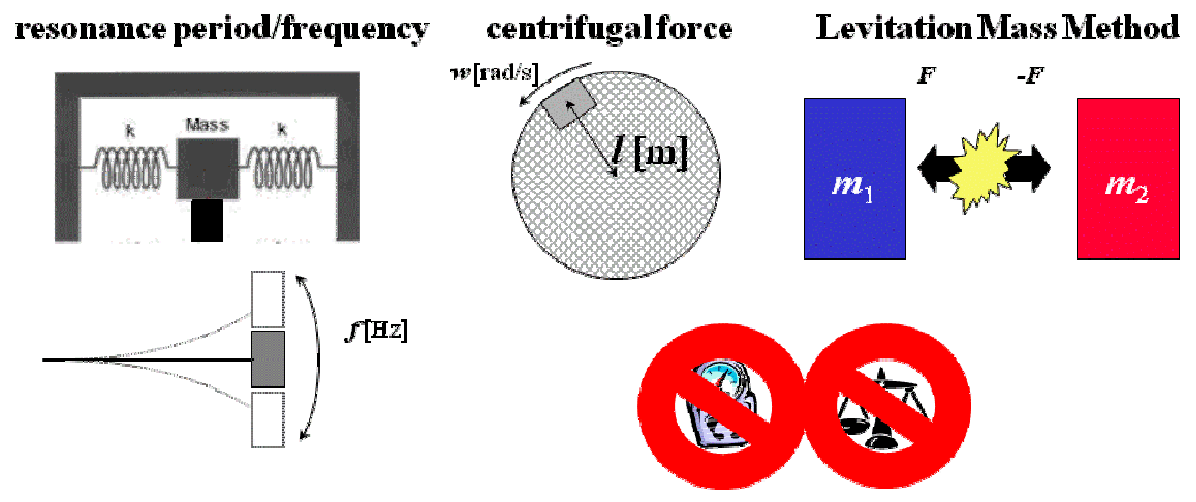

Fig. 10. Physical Principles for mass measurement on spacecraft.

Resonance period of mass-spring system was used on Skylab, Mir, and Spacelab.

\section{Thornton Body Mass Measurement Device (BMMD)}

The first operational body mass measurement system onboard spacecraft was the BMMD for Skylab program [2]. This was extensively tested on the ground [10]. The shape of the handholds were refined so that the most rigid posture could be assumed by a subject. The experiment was called M172. The BMMD is sometimes referred as M172 chair. Two units of Small Mass Measuring Device was also developed and flown to measure intake and output. This was called M074 experiment.
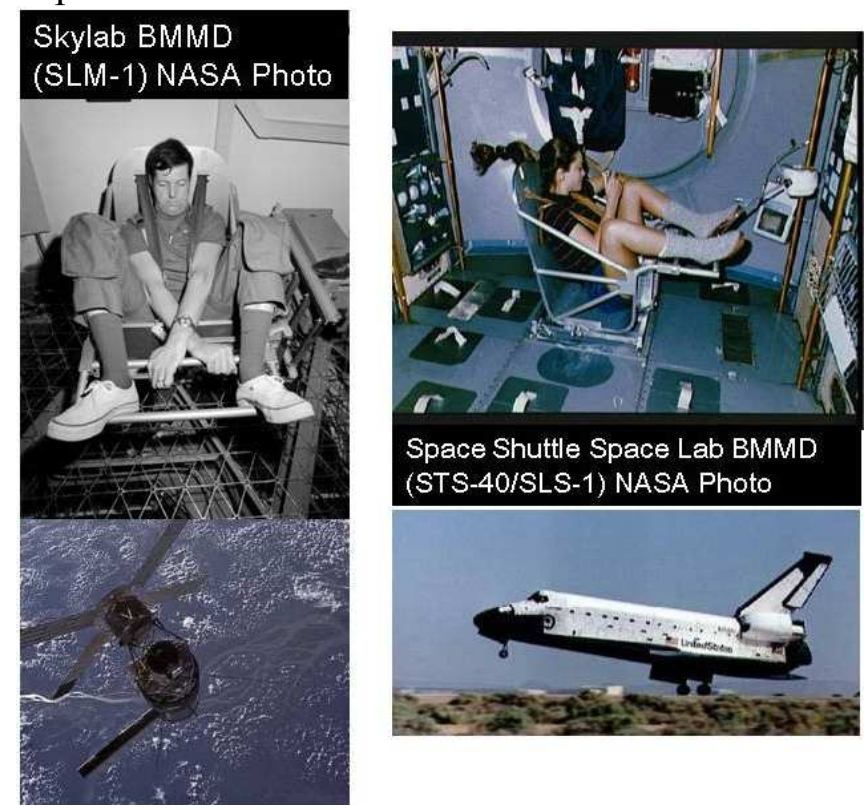

Fig. 11. Thornton Body Mass

Measurement Device.

Developed by Thornton [2], this high precision device was used for all Skylab onboard body mass measurements as well as for Space Shuttle - Spacelab lifescience missions.

It could accommodate calibration easily with known mass. The device was extensively tested by Thornton during SMEAT chamber simulation phase.

Retrofit ISS with this device is not easy, as this needs to be anchored. Float type of this device on a base may be achievable. 


\section{Russian MO-8 BMMD}

Russian BMMD for Soyuz and ISS program are basically the same design with mass-spring system principle [8]. This device is designated as an ISS medical operation system [9]. This means that this is an essential hardware to maintain astronaut/cosmonaut health onboard ISS. Weighing takes place every month for astronauts. Its procedure is called medical protocol MO-8 (Fig. 12). The device is fixed to the floor of a module, but can be tilted to save space. It takes a few minutes to deploy the device. Its post oscillates vertically. No calibration report became public of this BMMD. It is known to space medical community that this device has enough precision/uncertainty to track onboard body mass trend, but if its accuracy is up to its specification is not clear. It oscillates about 6 cycles for a run, and the results of 3 runs are averaged. The range of runs are known to be within about $0.5 \mathrm{~kg}$. It may have deviation in accuracy less than $2 \mathrm{~kg}$. The device depends on the spring constant of its specially manufactured metal spring. The service life of the spring is not known.
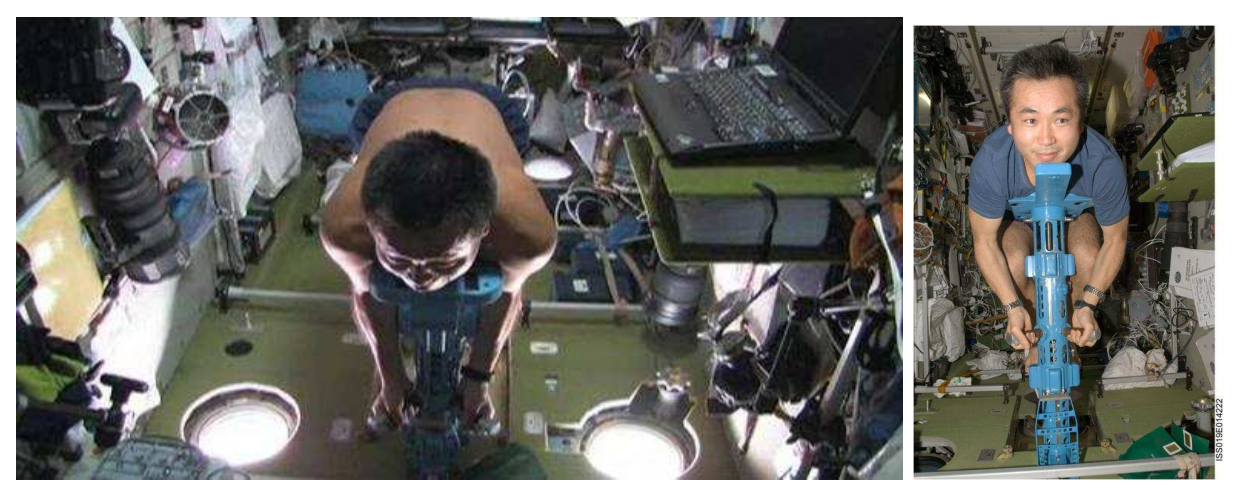

Fig. 12. Russian MO-8 BMMD on ISS.

Japanese astronaut Koichi
Wakata measures his body
mass in Russian ISS
Service Module, 2009.
The post oscillates
vertically.
Photo:NASA

\section{NASA SLAMMD}

As part of NASA's lifescience hardware suite, Space Linear Acceleration Mass Measurement Device (SLAMMD) (Fig.13) is installed to a Human Research Facility (HRF) rack [11]. Different from Russian BMMD, which does not need ground support, SLAMMD needs ground command support to activate the rack. As it is stowed with components detached, it takes 20 minutes to destow the device. It can be kept at a 'soft-stow' position with some protruded parts, from which it takes 10 minutes to deploy. Still astronauts need ground assistance to activate the HRF rack. This device uses leveraged arms to apply a light reference mass for calibration. This device is not known for its accuracy/precision. The result is the average of several runs, but their range seems to be over several $\mathrm{kg}$. Its accuracy is also questionable. As much as 6 to $7 \mathrm{~kg}$ deviation from a baseline value seems to have been experienced. Although there has not been a good comparison between SLAMDD and the Russian MO-8 BMMD, it is apparent to users that this device is not reliable, even for health status review purpose. Precise physiological experiments would not probably able to depend on its 2009 status of operation. This is not a portable device.

The operation of this device is somewhat complicated. One run consists of a unilateral movement of a subject from a distant position to a closer position, by a series of articulated arms. A subject clings to a holding jig during motion. A specially manufactured spring pulls the subject toward the rack face. Acceleration sensor measures subject motion. The body mass is derived from the spring constant and the acceleration value. The principle of measurement depends on the spring constant. 


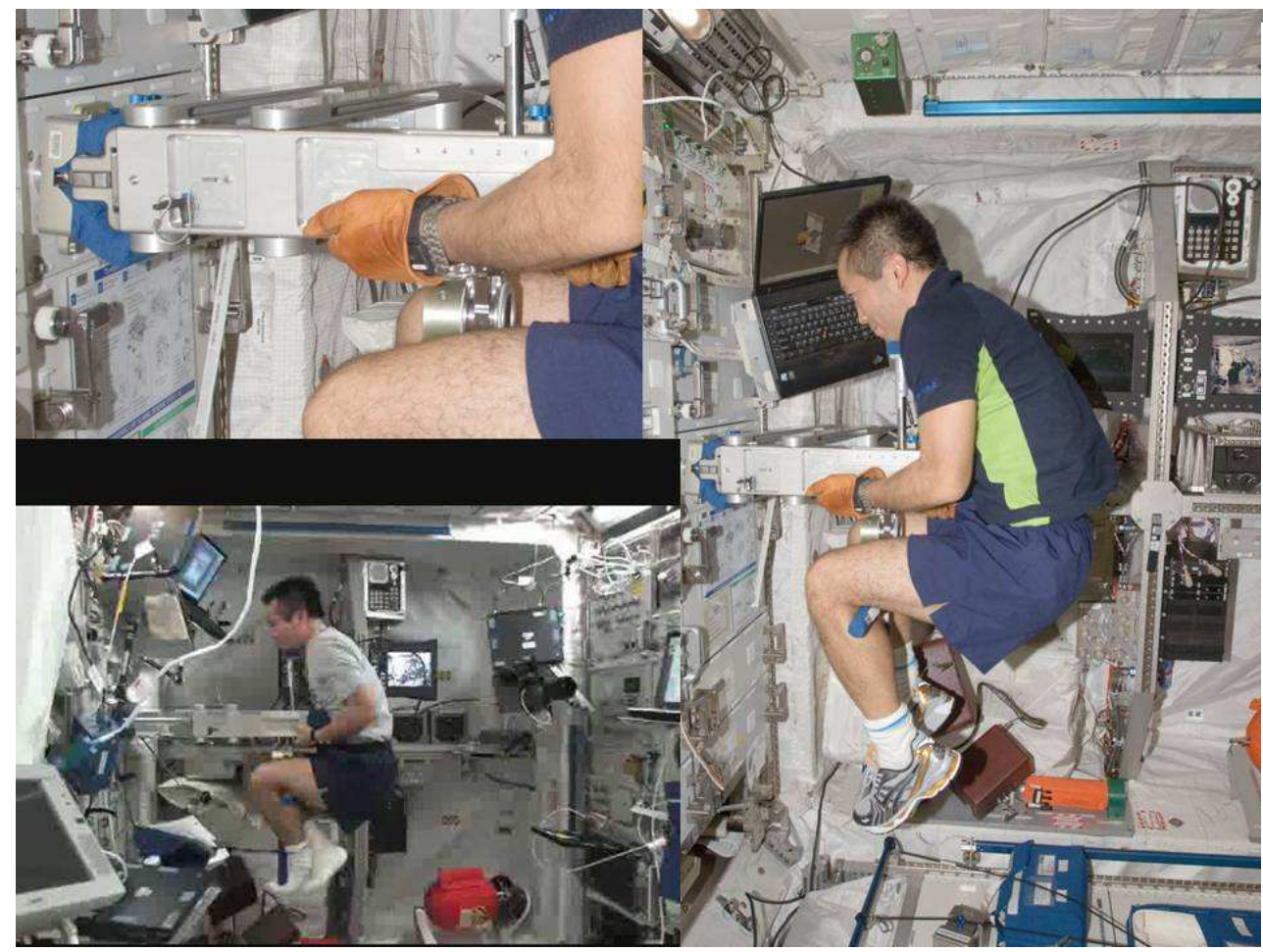

Fig. 13. NASA

SLAMMD.

Japanese astronaut

Koichi Wakata weighs

himself with the Human

Research Facility's

Space Linear

Acceleration Mass

Measurement Device

(SLAMMD) on ISS, 2009.

A special spring with a known spring constant in SLAMMD pulls the subject toward rack face via linked arms. Pull cable velocity is measured and subject body acceleration is calculated.

\section{Space Scale}

Finding storage space on ISS is becoming harder. Smaller, smarter operational and research hardware are sought. To materialize a portable and accurate body mass measurement system with current technology for ISS, Fujii and Shimada contemplated new principles for spacecraft use. First they evaluated applying Space Balance principle to human mass. Space Balance for small object is based on Levitation Mass method [5]. However, size of reference mass and elasticity of human body were concerns in applying Space Balance.

On Skylab, Space Shuttle, and Mir, BMMD's were anchored and used comparably small operational volume. ISS is huge, especially each module's longitudinal length is over $10 \mathrm{~m}$. The idea was that using longer translation path with smaller body acceleration will solve the problem of subject elasticity and voluntary posture change and improve measurement uncertainty (Fig. 14).

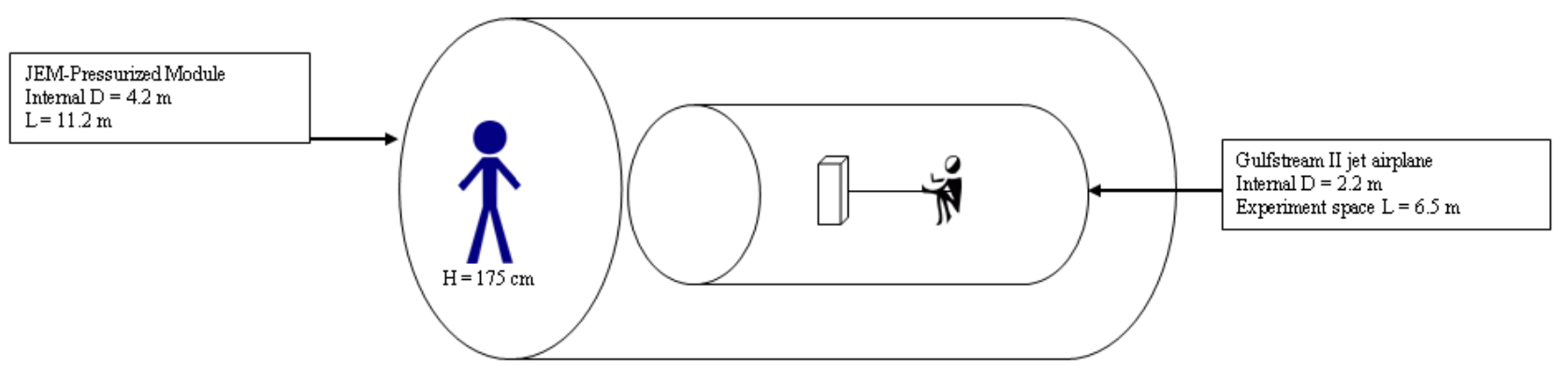

Fig. 14. ISS Kibo/JEM JPM module is large and long.

Comparison with a business jet Gulfstream-II gives an idea of its size.

Eventually, for ISS Space Scale prototype, rubber spring was chosen as a part for compactness and from safety standpoint. A desktop model was fabricated and its performance was measured (Fig. 15). A rubber spring with unknown spring constant pulls a subject. The force acting on the subject is measured directly by a force transducer in the pull cable system. Subject acceleration is measured by laser interferometry. Performance of the desk top model was promising [12]. 


\section{LMM (Space Balance) \\ Ground model study}

$\mathrm{m}=\mathrm{f} / \mathrm{a}$

rubber spring was selected

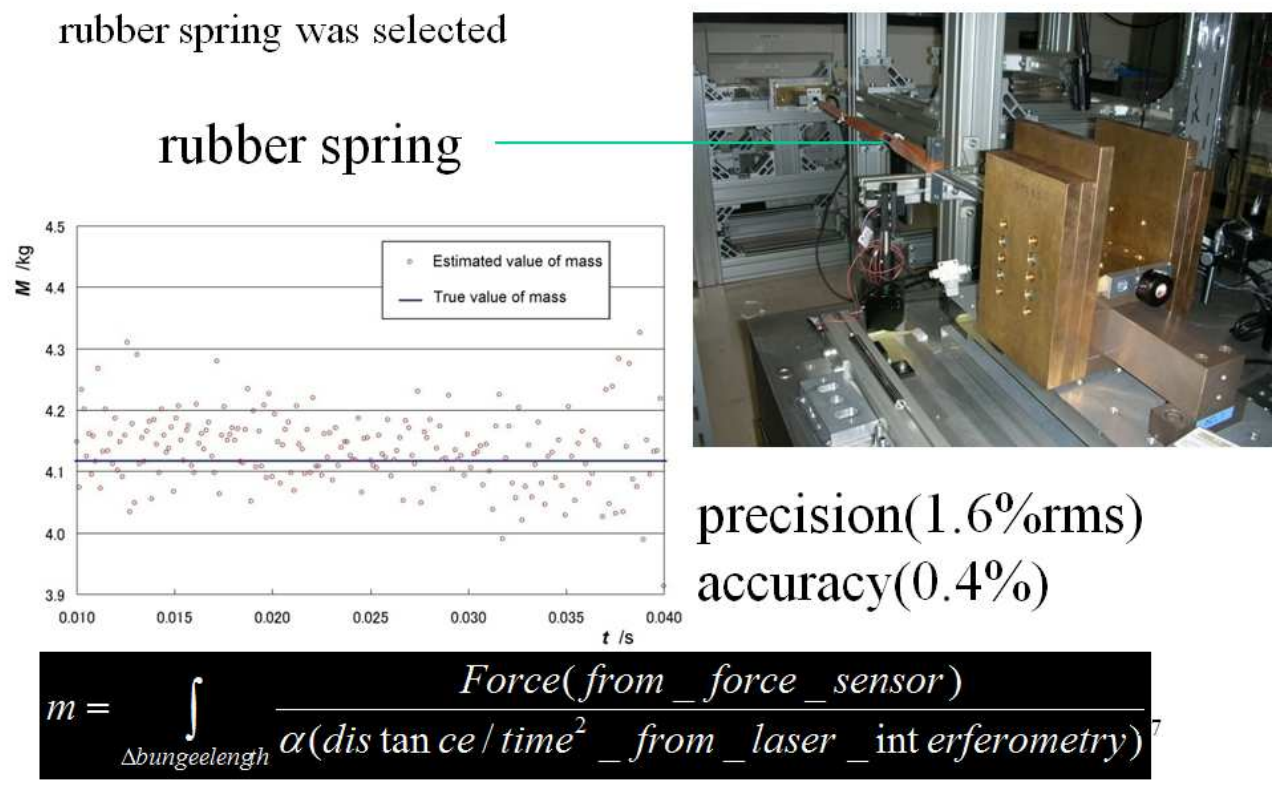

Fig. 15. Desk top prototype of Space Scale.

Rubber spring pull the dummy mass. The force acting on the mass is directly measured by a force transducer. Acceleration of the mass is measured by laser interferometry. Performance of this model was evaluated to be promising.

Next step following the desk top model was to flight test the system onboard a parabolic flight jet. Parabolic flight with a business jet gives maximum simulated weightlessness duration of 20 to 25 seconds. For our flight test, a 10 second-long trajectory was repeated (Fig. 16).

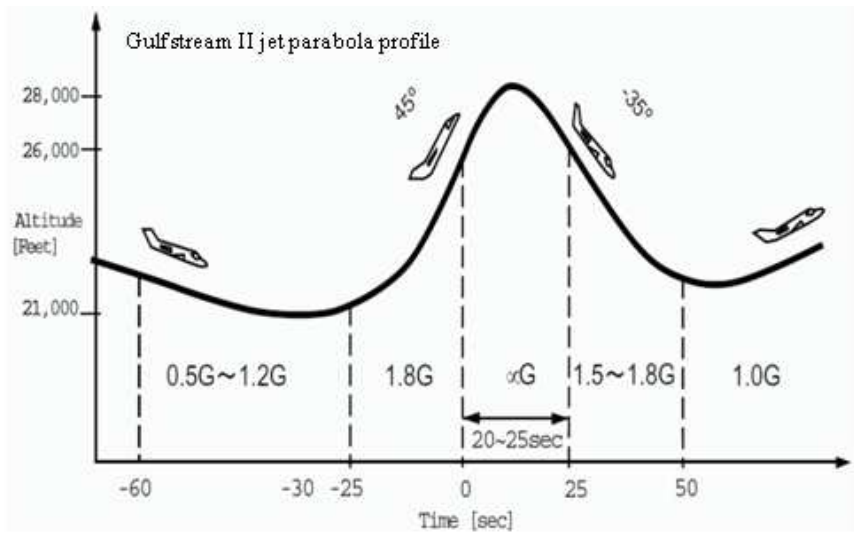

\section{$10+$ seconds of weightlessness}

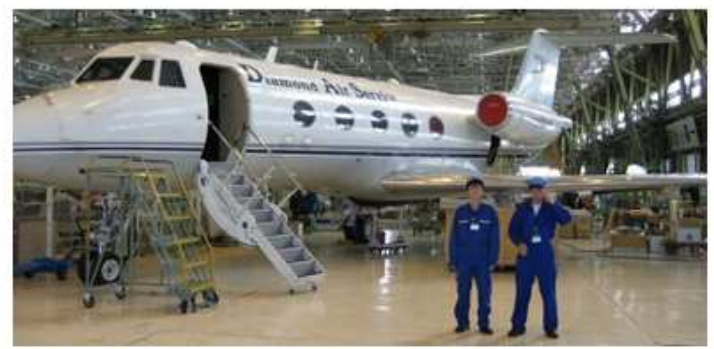

Fig. 16. Parabolic flight test profile for Gulfstream - II business jet.

The flight test instrumentation is shown in Fig. 17 and Fig. 18. Principle of instrumentation is the same as desk top experiment as in Fig. 15. The results were promising, as summarized below:

Total of 12 parabola data were submitted for analysis.

$>$ Bungee cord length change was $\sim 1 \mathrm{~m}$.

$>$ The real mass value of the dummy, $9.37 \mathrm{~kg}$, was measured to be $0.7 \%$ off by a mean, with standard uncertainty of $2.1 \%$.

$>$ Data reduction encountered difficulty with large residual G onboard the airplane. Space Scale performance in quiet orbital microgravity was not definitely tested.

After the dummy mass flight test, next step in Space Scale development was the subject flight test. Human flight test protocol was submitted to the Institutional Review Board of the Engineering Department, Gunma University, Japan. When the protocol was proposed, there was no IRB at the 
Engineering Dept. The authors had to start in asking for the establishment of an IRB at the department. After half a year of waiting, the protocol for the flight test was granted by the IRB.

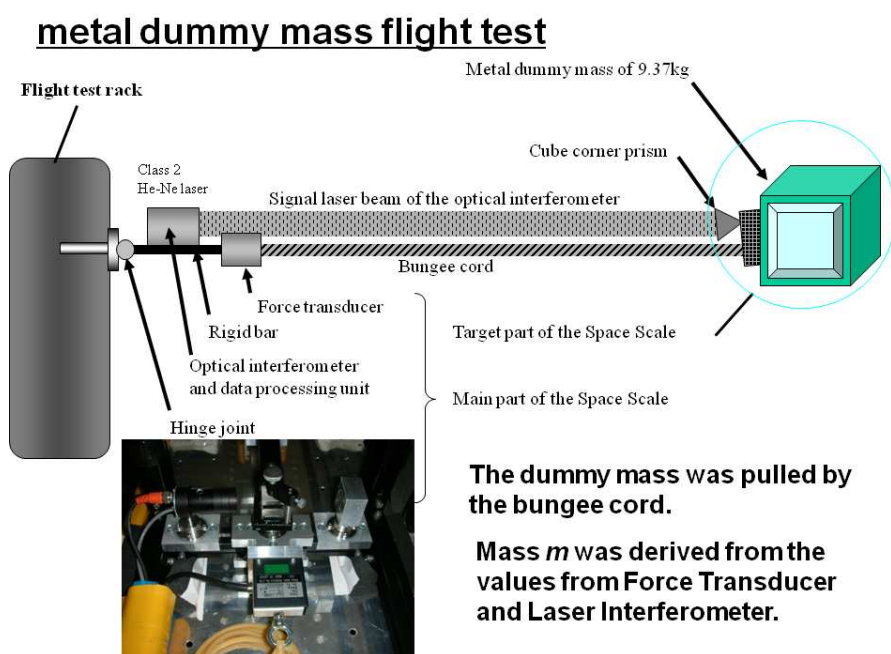

Fig. 17. Dummy mass flight test instrumentation.

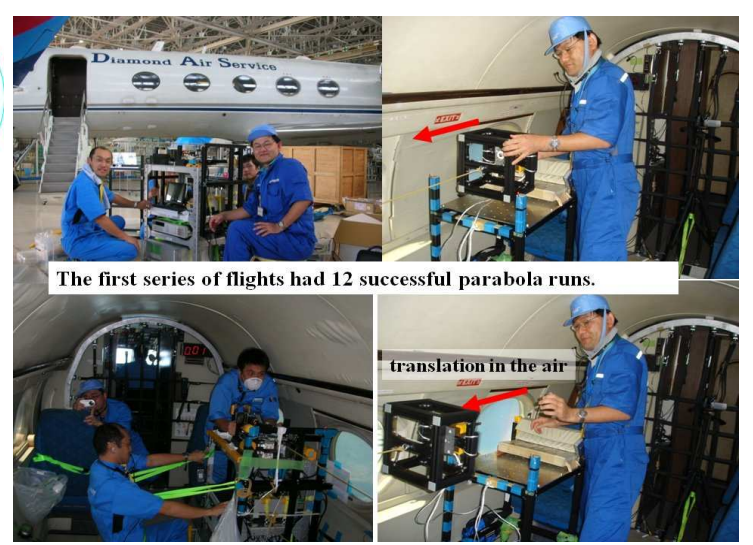

Fig. 18. Dummy mass parabolic flight test.

Layout for the flight test with a subject is shown in Fig. 19. Due to residual G noise in the cabin, data analysis was difficult. With noise mitigation software, one run yielded uncertainty of $1 \%$.
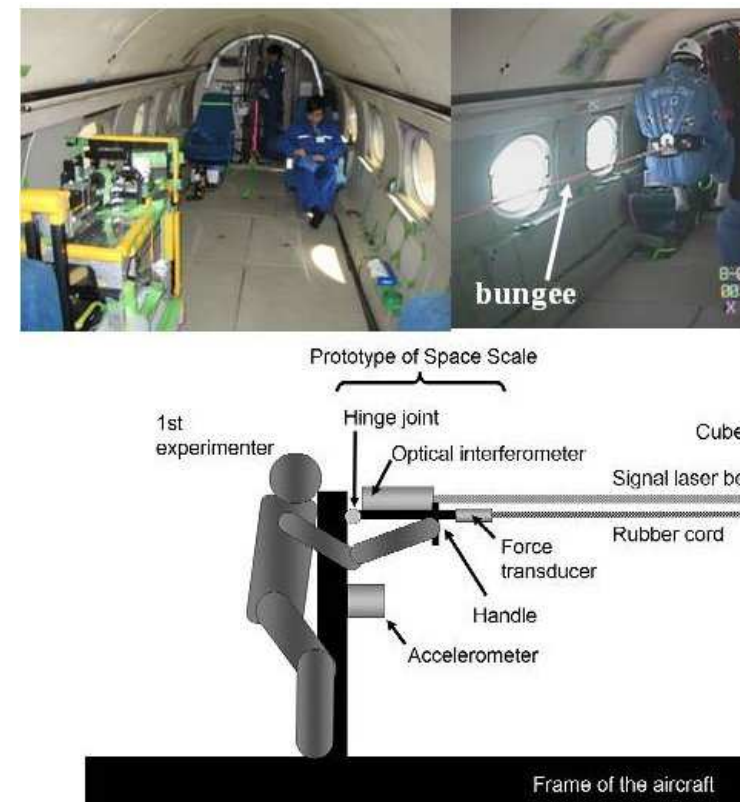

Hinge joint

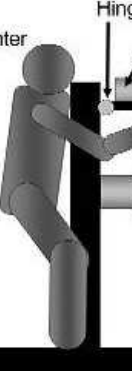

Optical interferomete

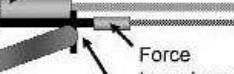

Force
transducer

Handle

Accelerometer

(n)

r

Frame of the aircraft

Results from subject parabolic flight is summarized in Fig.20. What found was that residual acceleration in the cabin, $G$ noise, was too large to assess the performance of the flight test model Space Scale. Along the longitudinal axis of the rubber spring, approximately $4 \times 10^{-2} \mathrm{~ms}^{-2}$ was the $\mathrm{G}$ noise level. Extensive data analysis was tried $[13,14]$, but only one run data were recovered.

The authors left the parabolic flight test with unanswered question: if effects from elasticity of the body and voluntary movement of the subject are small enough to warrant design of Space Scale? As parabolic flight tests does not seem to be usable for this validation, the authors turned to ISS itself.

Japanese astronaut Koichi Wakata, Ph.D. had been to fly as the first Japanese long duration ISS crewmember in 2009. We had a chance to discuss the issue of ISS body mass measurement. He recognized the issue, and became interested if a crewmember can be quietly towed by a bungee cord. 
He self-videotaped his voluntary science session during his free time. Fig. 21 shows body pitching tendency when the tow point is at the head. When pulled at a few centimeters above the navel, posture of the crewmember stayed constant. Fig. 22 shows difference in spring stretch. Both Fig. 21 and Fig. 22 is a still capture from a motion picture.

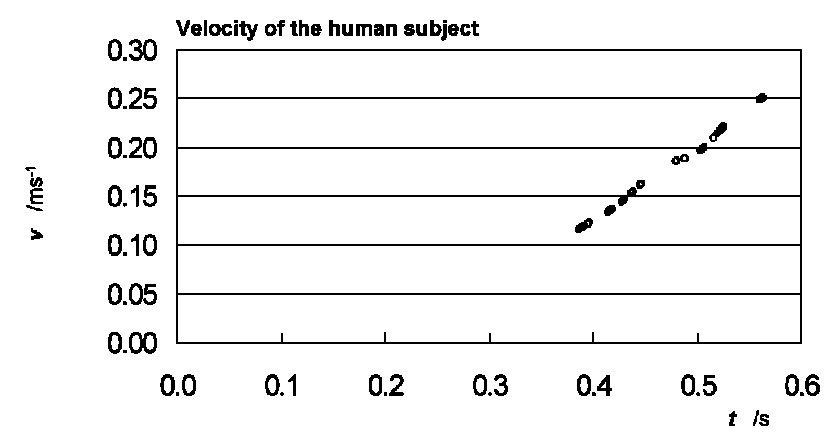

Fig. 20A (above)

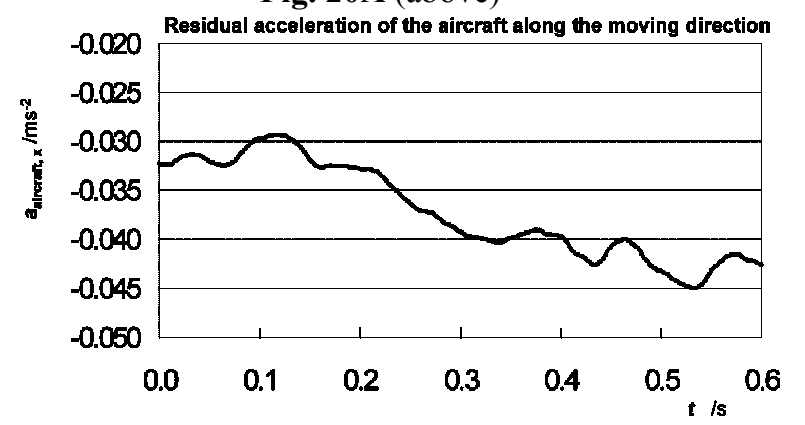

Fig. 20C

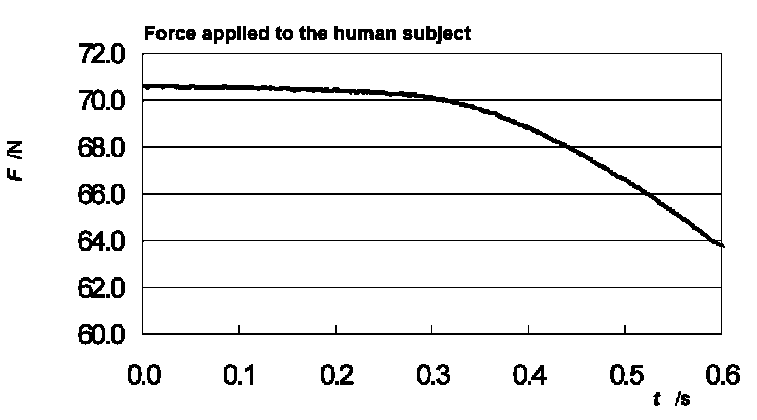

Fig. 20B

Fig. 20 A B C. Human subject parabolic flight test results with airplane model Space Scale.

Due to a large residue $\mathrm{G}$ noise in the airplane cabin $\left(\sim 4 \times 10^{-2} \mathrm{~ms}^{-2}\right)$, only one run yielded mass estimate, whose uncertainty was $1 \%$ of the real mass. Subject velocity was $\sim 0.2 \mathrm{~ms}^{-1}$. Bungee cord tension was $\sim$ $70 N$. Subject acceleration was smooth and small, its value around $5 \times 10^{-3} \mathrm{G}$.

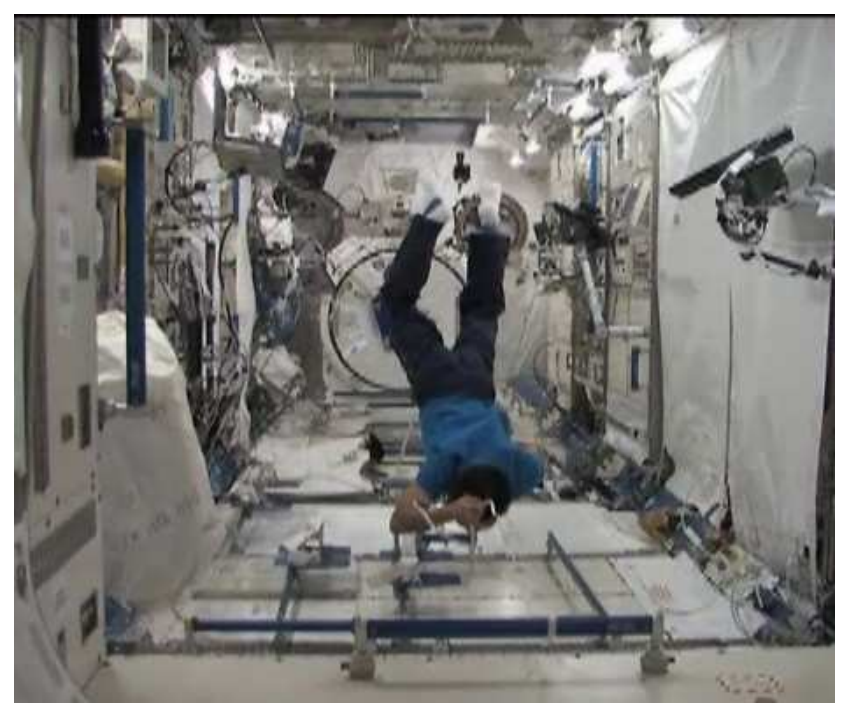

Fig. 21 A. Subject towed at the head.

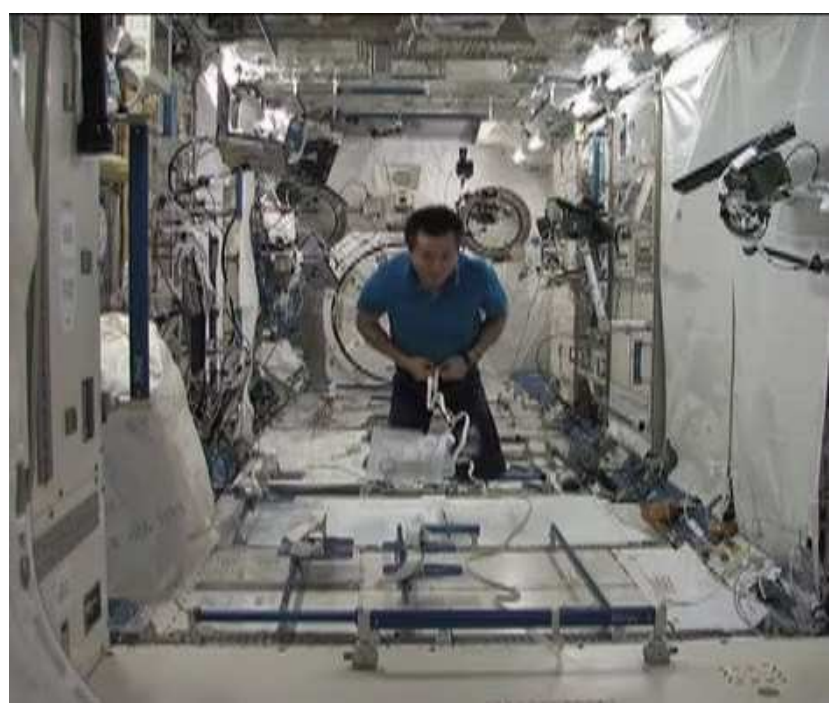

Fig. 21B. Subject towed at above the navel.

A weak rubber spring (rubber tape used for pajamas, etc.) was used for the demonstration. When the subject was towed by the extended rubber spring at the head (Fig. 21A), head-pitch-down motion was induced, which distorted whole posture. It was unrecoverable. When tow point was a few centimeters above the navel (Fig. 21B), where body's center of mass is, crewmember's posture was stable with a small amount of head-pitch-down tendency. 


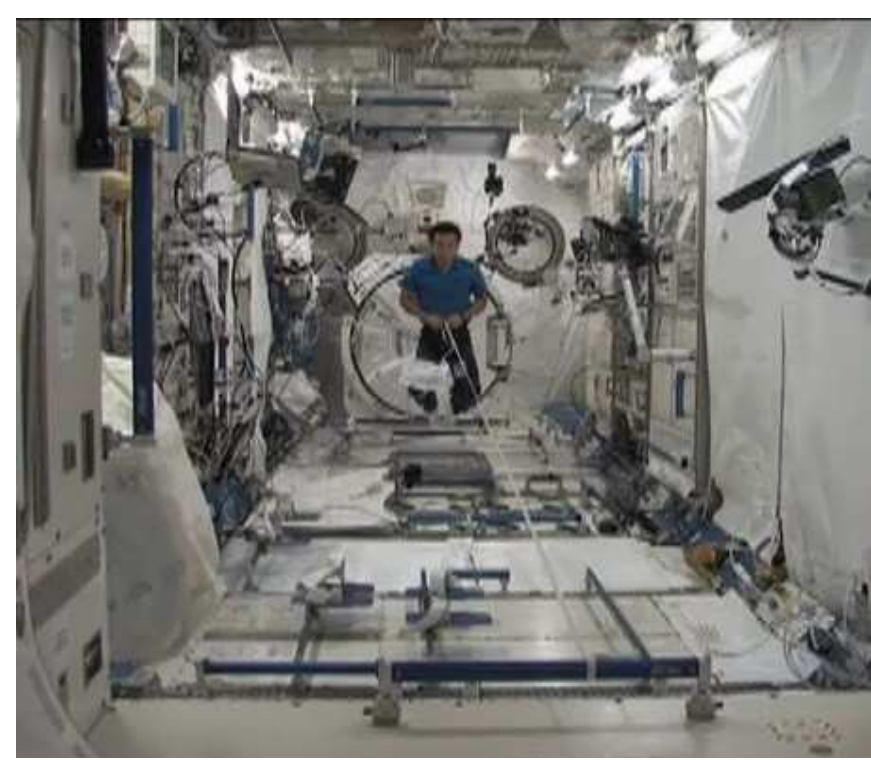

Fig. 22A. Rubber cord extended to $6 \mathrm{~m}$.

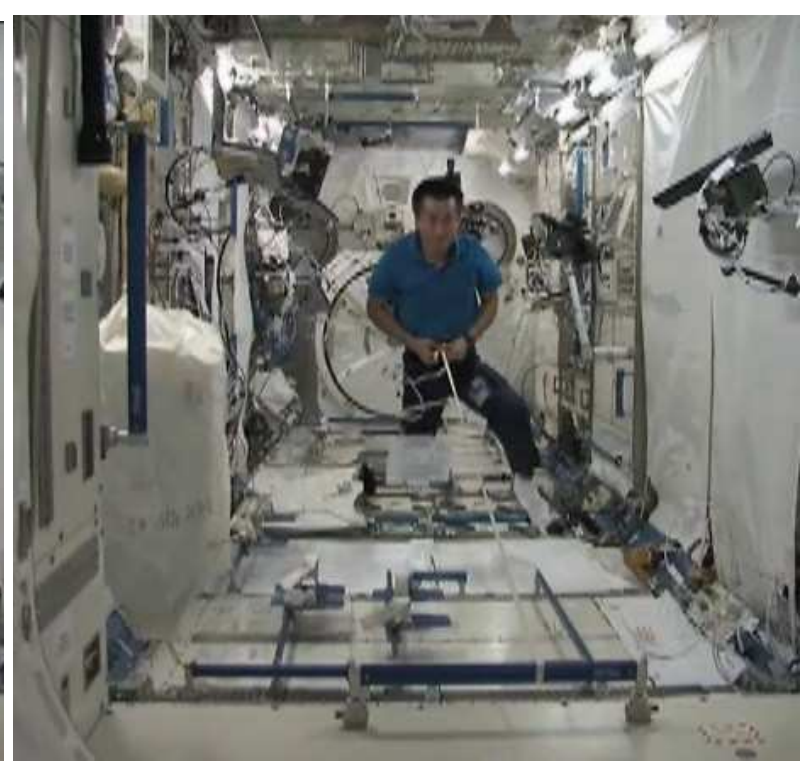

Fig. 22B. Rubber cord extended to $3 \mathrm{~m}$.

Fig. 22A and Fig.22B demonstrates stability of subject posture and body orientation. The same rubber spring was stretched to $6 \mathrm{~m}$ (Fig. 22A) and $3 \mathrm{~m}$ (Fig. 22B). As both cases used navel tow point, not much difference in posture stability was found visually. Posture stability was excellent in both cases.

\section{Thornton “Space Drop Tower” by Linear Artificial Gravity}

When artificial gravity on ISS is discussed, it is always about artificial gravity by centrifugal force. However, as indicated by Thornton [2], a few seconds of linear artificial gravity can be obtained onboard ISS. This period is long enough for the inner ear to react to. Thus this is a good segway to new vestibular research. From the point of body mass measurement, if quality linear acceleration field is achieved, ground load cell or balance can be used in the spacecraft. Then all problems associated with calibration can be neglected. Sensing linear acceleration can be done by Space Scale components. In that sense, Space Drop Tower could be an extension of Space Scale. This is a space station unique facility, as it needs a long translation path for a subject (Fig. 23).

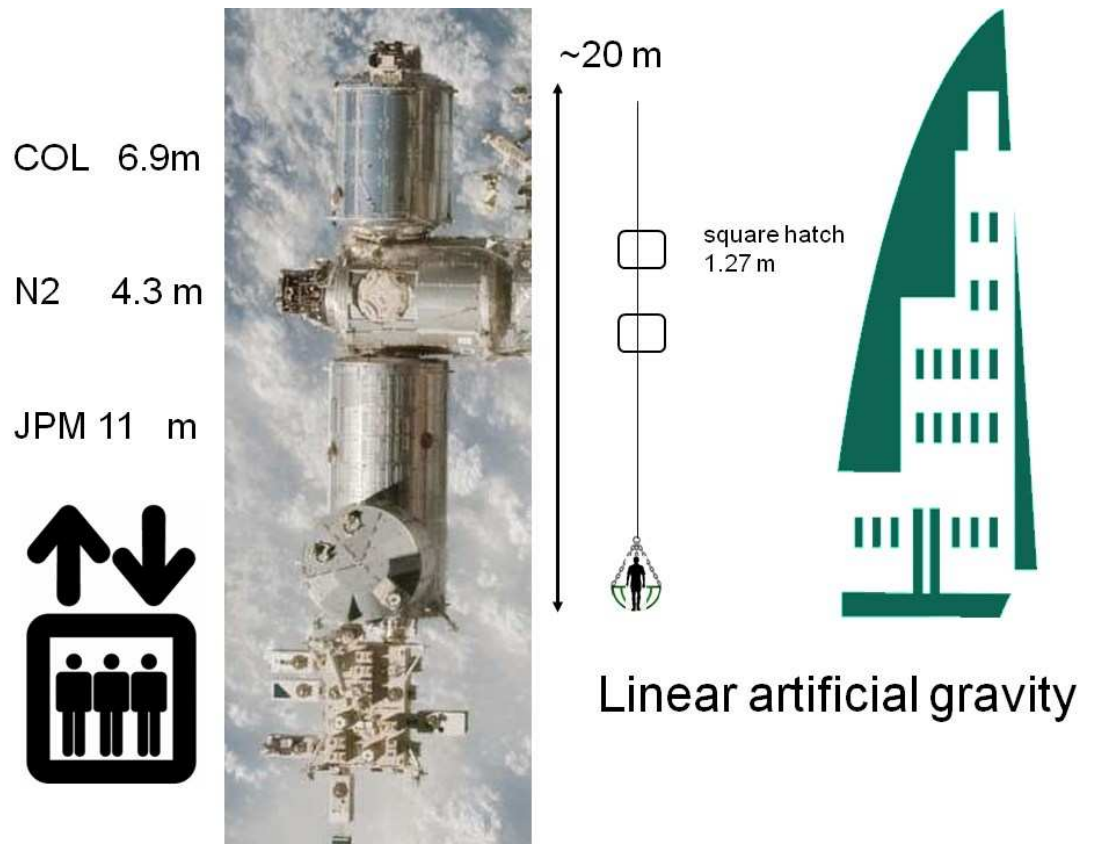

Fig. 23. Thornton Space Drop Tower by Linear Artificial Gravity.

Longitudinal path from Kibo-JPM module to Columbus module is almost $20 \mathrm{~m}$ long. This is equivalent to an elevator in a 7-story high building. Hatches connecting the modules are square and large enough for a person and a carrier basket.

This dimension of Drop Tower can generate a few seconds of $0.1 \mathrm{G}$ acceleration $\left(0.98 \mathrm{~ms}^{-2}\right)$. How to halt the basket would be the most difficult safety question; however, it is expected that maybe up to $4 \mathrm{~ms}^{-1}$ the basket velocity may be controlled manually or by body movement. 


\section{Discussion}

Current body mass measurement system onboard ISS are bulky, not portable, and depends on special spring's constant. Neither they are with a good proven accuracy/precision. As ISS becomes more and more crowded, rack-mounted hardware are requested to be substituted by supplies. Smarter, smaller and lightweight hardware are needed.

The principle of lengthy translation by the tow using a weak spring has many advantages for the body mass measurement on ISS. Longer translation means better accuracy by integration. Space Scale can be materialized as a small hardware which is highly portable, such as Space Scale. When a time comes for spring replacement for Russian BMMD, replacing it with something not fixed to the floor of the Service Module should be considered. ISS system service life is 2028, and International Partners are heading for ISS usage by 2020. Space Drop Tower and Linear Artificial Gravity is a good topic for planning the next phase of ISS research.

\section{Acknowledgements}

The authors appreciate Dr. Wakata's dedication of his time for video filming during the busy hours on ISS. We also thank Dr. Thornton, whose guidance lead us to many possible options.

\section{References}

[1] W. E. Thornton, J. Ord: Physiological Mass Measurement in Skylab. in: Biomedical Results From Skylab. NASA SP-377, 1sda.jsc.nasa.gov/gooks/skylab/skylab_toc.cfm (1977) p. 175-182

[2] W. Thornton, Y. Fujii ,K. Shimada: Mass Measurement in Space, 1964-2010, (in this issue)

[3] S.M. Smith, S. R. Zwart, G, Glock, et al.: The Nutritional Status of Astronauts Is Altered after Long-Term Space Flight Aboard the International Space Station. J. Nutr. 135 (2005), p. 437-443

[4] A. Rivetti, G. Martini, F. Alasia, et al.: BIC 3, the latest inertial centrifugal balance for mass measurement in weightless conditions. Microgravity Science and Technology, Vol.20 (1) (2008) p.7-15

[5] Y. Fujii, H. Fujimoto, R. Watanabe, Y. Miki: Balance for measuring mass under microgravity conditions. AIAA Journal, Vol. 39(3) (2001) p. 455-457

[6] Y. Fujii, K. Shimada, K. Maru: Instrument for measuring the body mass of astronauts under microgravity conditions. Microgravity Science and Technology. Vol.22(1) (2010) p. 115-121

[7] K. Shimada, Y. Fujii : Parabolic flight test of SPACE SCALE - new body mass measurement device by laser interferometry. Aviat Space Environ Med. ; 79 (3): (2008) p.216

[8] V.A. Sarychev: Device for mass measurement under zero gravity conditions.Acta Astronautica, VOl.7 (1980) p. 719-730

[9] Y.E. Nikoloevna: ISS Russian Medical Hardware Catalog. (2009) p. 41-53

[10] D. Hitt, O.K. Garriott, J.P. Kerwin: Homesteading Space: The Skylab Story. Univ. of Nebraska Press, ISBN978-0803224346. (2008)

[11]D.C.Smith, K.A. Kaufman: Space Linear Acceleration Mass Measurement Device (SLAMMD) for the Human Research Facility (HRF). SAE Technical Papers. No. 981652 (1998)

[12]K. Shimada, Y. Fujii: Reconsideration of body mass measurement on the International Space Station and beyond. Key Engineering Materials, Vol. 381-382, (2008) p. 461-464

[13]T. Mizuno, T. Adachi, M. Takasaki, Y. Ishino: Mass Measurement System Using Relay Feedback with Hysteresis. J System Design and Dynamics. Vol.2 (1) (2008) p.188

[14]T. Ono, H. Uozumi, O. Honda, K. Nagata: Mass-measurement under weightless conditions by the frequency-controlled method. Measurement. Vol.22 (1997) p.87-95 